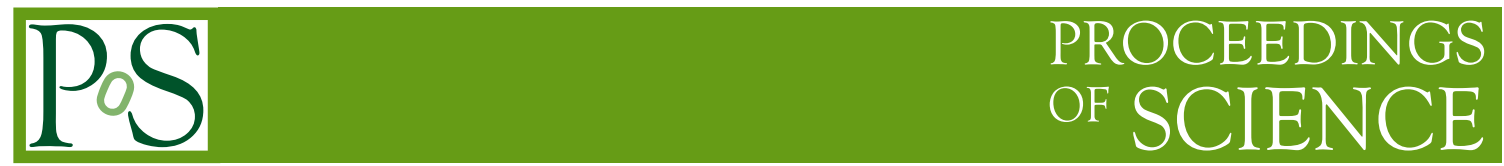

\title{
Precision QCD measurements from CMS
}

\author{
Salim Cerci $^{a, b, *}$ \\ ${ }^{a}$ Adiyaman University, \\ Faculty of Arts and Sciences, Department of Physics, 02040 Adiyaman, Turkey \\ ${ }^{b}$ On behalf of the CMS Collaboration \\ E-mail: Salim.Cerci@cern.ch
}

Recent measurements of jet events are presented which test QCD with unprecedented precision. The measurements are based on Run 2 data collected with the CMS detector at the LHC. The data are compared to various theoretical predictions and Monte Carlo event generators after correcting for detector effects. Such studies can improve experimental understanding of jets and jets substructure.

The Ninth Annual Conference on Large Hadron Collider Physics-LHCP2021

7-12 June, 2021

online

*Speaker

(c) Copyright owned by the author(s) under the terms of the Creative Commons 


\section{Introduction}

In high-energy proton-proton collisions, processes governed by the strong interaction are dominant. Quantum chromodynamics (QCD), the gauge theory describing the strong interaction between quarks and gluons, has a great testing ground at the LHC, providing rich jet data in a previously unexplored region. In this document, recent jet related measurements performed with the data collected by the CMS [1] experiment are presented.

\section{Multijet correlation measurement}

Multijet events and the modeling of their characteristics play an important role in precision measurements, as well as in searches for new physics. The understanding of the structure of multijet final states is therefore crucial for analyses of those events. A recent measurement has been published by CMS [2] related to collinear (small-angle) and large-angle radiation as well as momentum distributions in multijet production. In the analysis two different topologies of three-jet events and $\mathrm{Z}+2$ jet events are used. Events are categorized by using two observables, one of which is the transverse momentum ratio to distinguish the soft from hard radiation and the second one is the angular separation to define a collinear and a large-angle radiation. Reasonable agreement with parton shower and matrix element calculations is observed within the available phase space, except the hard radiation region $\left(p_{T 3} / p_{T 2}>0.6\right)$. In Figure 1 , the $p_{T 3} / p_{T 2}$ distribution is shown for the angular radiation of $\Delta R_{23}$.
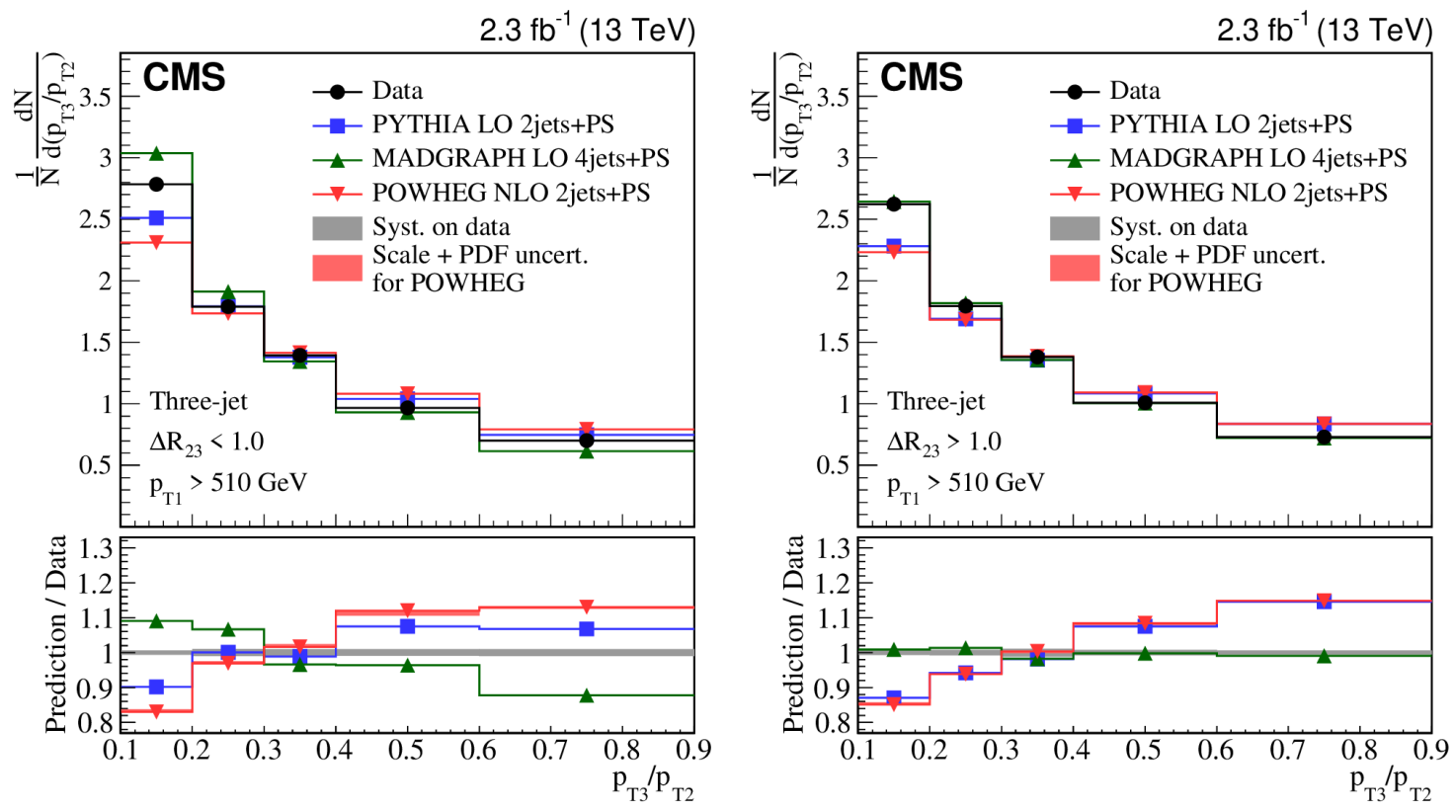

Figure 1: Comparison of $p_{T 3} / p_{T 2}$ for three-jet events with the theoretical predictions for small-angle radiation $\left(\Delta R_{23}<1.0\right)$ (left) and for large-angle radiation $\left(\Delta R_{23}>1.0\right)$ [2]. 


\section{Inclusive jet production dependence on distance parameter}

The anti- $k_{T}$ algorithm, the commonly used jet clustering algorithm at CMS, requires the distance parameter $R$ which corresponds to the radius of the jet in the $y-\phi$ plane, where $y$ and $\phi$ are the rapidity and azimuthal angle, respectively. The amount of energy clustered into the jet is controlled by the distance parameter $R$ and various contributions having dependence on $R$. The measurement of a jet production cross section as a function of $R$ gives the opportunity to examine the theoretical modeling of the perturbative and nonperturbative (NP) effects in parton evolution. The CMS Collaboration has recently published [3] the inclusive jet cross section measurement for each $R$ parameter, relative to $R=0.4$, covering $R=0.1-1$ in intervals of 0.1 . The unfolded results are compared to leading order (LO) and higher order (NLO, NNLO) predictions with different parton shower and with and without NP corrections. In Figure 2, the ratio of differential cross sections of inclusive jets with respect to AK4 jets is shown. NLO prediction using POWHEG+PYTHIA [4, 5] (CUETP8M1 tune) describes the data at high $p_{T}$ however some deviations at low $p_{T}$ are observed for larger jet radii.

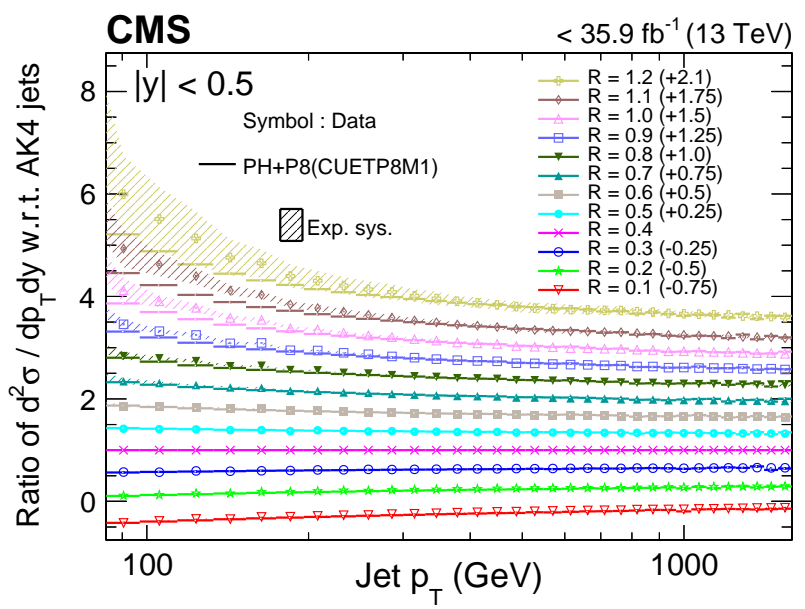

Figure 2: Ratio of the differential jet cross sections for different sizes with respect to AK4 jets in the region $|y|<0.5[3]$.

\section{4. $\mathrm{Z} / \gamma+$ jet cross section measurements}

The differential cross sections of $\mathrm{Z}+$ jets and $\gamma+$ jets production and their ratio are measured as a function of the vector boson $p_{T}$ by using the data recorded by the CMS experiment [6]. In order to explicitly study the collinear $\mathrm{Z}$ emission, the $\mathrm{Z}+\mathrm{jet}$ cross section is also given as a function of the distance between the $\mathrm{Z}$ boson and the closest jet. Due to the sensitivity of higher order electroweak corrections at high boson $p_{T}$ region, $\mathrm{Z} / \gamma$ ratio plays a crucial role for the background estimations in beyond SM physics searches. In the analysis, the vector boson is required to have $p_{T}>200 \mathrm{GeV}, \eta$ or $|y|<1.4$. The unfolded data is compared with predictions from LO and NLO calculations from MADGRAPH5 aMC@NLO, as well as the NLO QCD+EW calculation from SHERPA + OPENLOOPS. Furthermore, a comparison of $\gamma+$ jets measurement with with NLO JetPhox predictions is performed. Figure 3 shows the comparison of differential cross section ratio 
of $\mathrm{Z}+$ jets to $\gamma+$ jets as a function of the vector boson $p_{T}$ with the theory predictions. Overall, a good agreement is observed between the data and the theory for both the $\gamma$ and $\mathrm{Z}$ boson final states, although in some regions of phase space a few tens of percent deviations are observed. In general, the perturbative NLO corrections exhibit a better agreement with the measurements.

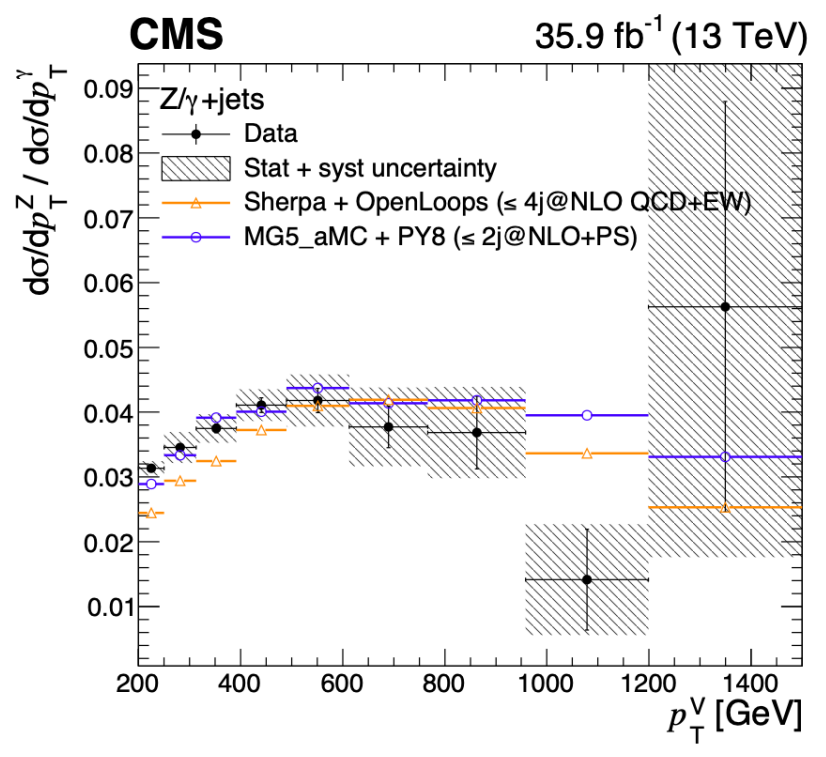

Figure 3: Comparison of differential cross section ratio of $\mathrm{Z}+$ jets to $\gamma+$ jets as a function of the vector boson $p_{T}$ with the theory predictions [6].

\section{References}

[1] CMS Collaboration, The CMS Experiment at the CERN LHC, 317 JINST 3, (2008) S08004. doi:10.1088/1748-0221/3/08/S08004.

[2] CMS Collaboration, Measurements of angular distance and momentum ratio distributions in three-jet and Z+two-jet final states in pp collisions, Accepted by EPJC, arXiv:2102.08816 (2021).

[3] CMS Collaboration, Dependence of inclusive jet production on the anti- $k_{T}$ distance parameter in pp collisions at $\sqrt{s}=13 \mathrm{TeV}$, JHEP 12, 082 (2020). doi:10.1007/JHEP12(2020)082

[4] S. Frixione, P. Nason, and C. Oleari, Matching NLO QCD computations with Parton Shower simulations: the POWHEG method, JHEP 11, 070 (2007). doi: 10.1088/11266708/2007/11/070

[5] T. Sjostrand et al., An introduction to PYTHIA 8.2, Comput. Phys. Commun. 191 (2015) 159. doi: 10.1016/j.cpc.2015.01.024

[6] CMS Collaboration, Measurements of the differential cross sections of the production of Z+jets and $\gamma+j e t s$ and of $Z$ boson emission collinear with a jet in pp collisions at $\sqrt{s}=13 \mathrm{TeV}$, JHEP 05 (2021) 285. 